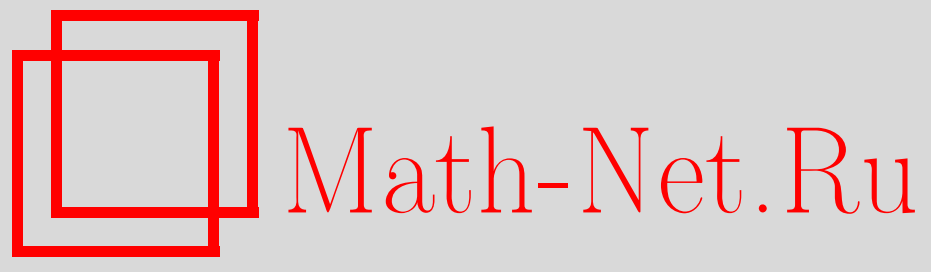

В. П. Маслов, Об одном новом ранговом распределении, Матем. заметки, 2006, том 80, выпуск 3, 469-470

DOI: https://doi.org/10.4213/mzm2834

Использование Общероссийского математического портала Math-Net.Ru подразумевает, что вы прочитали и согласны с пользовательским соглашением http://www . mathnet.ru/rus/agreement

Параметры загрузки:

IP: 54.224 .60 .19

26 апреля 2023 г., 08:08:34

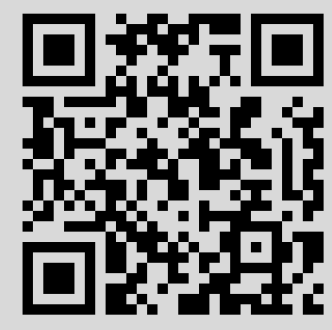




\section{ОБ ОДНОМ НОВОМ РАНГОВОМ РАСПРЕДЕЛЕНИИ}

\section{В. П. Маслов}

В ранговых распределениях, полученных автором, неизвестными (или подгоночными) являются четыре параметра:

- температура T;

- параметр Ципфа или размерность D;

- максимальный ранг $r_{\max }=N \gg 1$ и

- параметр лакунарности или префактор [1].

Если существует соотношение между этими параметрами, то мы по аналогии с термодинамикой будем называть эти соотношения уравнениями состояний. Два из этих параметров $T$ и $N$ уже содержатся в формулах распределения Бозе-Эйнштейна, а два другие - в законе Ципфа.

В ранговых распределениях участвуют только целые числа - ранг (номер) $r$ объекта и число встречаемости $\omega_{r}$ объекта: задается ранг и ему сопоставляется число встречаемости. Если разность между ближайшими числами встречаемости $\omega_{l}$ и $\omega_{l+1}$ больше единицы, то говорят, что имеется лакуна с $\omega_{l}-\omega_{l+1}-1$ точками. Мы получаем ранговые распределения из обобщенного распределения типа Бозе-Эйнштейна для частиц [2], сопоставляя частицам объекты более общего типа (например, слова в частотном словаре) и учитывая, что сумма всех частиц на уровнях $\lambda_{i}>\lambda_{l}$ есть ранг, отвечающий $\lambda_{l}$.

В исходных положениях распределения Бозе-Эйнштейна определяется число частиц, находящихся на данном уровне энергии $\lambda_{i}$. Однако согласно формуле Бозе-Эйнштейна [3] это число не будет целым. Это означает, что, например, если оно много меньше единицы, то на достаточно большом интервале энергий $\lambda_{i}$ находится только одна частица. Значит, согласно формуле Бозе-Эйнштейна имеются уровни энергии $\lambda_{i}$, на которых нет частиц, что в свою очередь означает наличие значительных лакун с точки зрения теории рангов.

Постановка вопроса в формуле Бозе-Эйнштейна противоположна точке зрения обычного рангового распределения. В постановке задачи для распределения Бозе-Эйнштейна, наоборот, заданы уровни энергии и находится число частиц, которые им соответствуют.

Набор чисел встречаемости $\left\{\omega_{l}\right\}$ принадлежит набору целых чисел $\{i\}$, но мы не можем знать, какие точки лакун соответствуют уровням энергии, на которых "сидит" нуль частиц, а какие точки лакун нужно аннулировать - им не отвечают никакие уровни энергии. Положим $\lambda_{i}=\left[i+\alpha i^{1+\gamma}\right]$ (ангармонизм), где $\alpha$ и $\gamma$ - подгоночные константы. Мы не требуем, чтобы набор $\left\{\omega_{l}\right\}$ принадлежал набору $\left\{\lambda_{i}\right\},-$ мы находим эти константы, сопоставляя окончательную теоретическую кривую, полученную на основе доказанного автором обобщенного распределения типа Бозе-Эйнштейна [4]-[6], с экспериментальными значениями $\omega_{l}$.

В работе [6] мы предложили психологическую интерпретацию как функции предпочтения этой детерминистической лакунарности вида $\alpha \omega^{1+\gamma}$, аналогичную той, которая легла в основу нелинейного среднего [7].

Итак, в детерминистической лакунарности мы аннулируем ряд целых точек $i$, а из формулы Бозе-Эйнштейна мы находим, что некоторым из чисел $\lambda_{i}$ соответствует пустое множество объектов (например, нуль слов в массиве текстов). 
Если $T \sim 1$, то при $\omega \rightarrow \infty$ из [4; теорема 1] следует новое ранговое распределение

$$
\begin{aligned}
r & =\sum_{\omega}^{\omega_{\max }} \frac{c}{e^{\beta\left(i+\alpha i^{\gamma+1}\right)}-1} \sim \int_{\omega}^{\infty} \frac{c}{\left(e^{\beta\left(x+\alpha x^{\gamma+1}\right)}-1\right)\left(1+\alpha(1+\gamma) x^{\gamma}\right)} d x \\
& \sim \int_{\omega}^{\infty} e^{-\beta x-\alpha x^{1+\gamma}}\left(1+\alpha(1+\gamma) x^{\gamma}\right)^{-1} d x
\end{aligned}
$$

$c=$ const. Отсюда при $\omega \rightarrow \infty$

$$
\log r+c_{1} \omega^{1+\gamma} \sim 0 .
$$

Константы $\gamma$ и $c_{1}$ могут быть определены по тем же программам, по которым это делается для распределения Ципфа.

Отметим, что при $\gamma=0$ оно совпадает с ранговым распределением Гусейн-Заде [8], построенным на основе других соображений и для ситуации $N \sim 1$.

Заметим в заключение, что ранее автором было показано, что при условии $T \gg 1$ формула Бозе-Эйнштейна переходит в формулу (6) работы [4] тривиальным вычислением, а эта формула, в свою очередь, при $\omega \rightarrow \infty$ переходит в закон Ципфа. Именно, ранг $r$ удовлетворяет соотношению, аналогичному [5], [6]:

$$
\frac{r}{r_{\max }}=(1+\gamma) \ln \left(1+\frac{1}{\alpha(\gamma+1) \omega^{\gamma}}\right)-\ln \left(1+\frac{1}{\alpha \omega^{\gamma}}\right),
$$

где $\omega$ - частота встречаемости объекта ранга $r$, и $r \sim T /\left(\gamma \alpha \omega^{2 \gamma}\right)$ при $\omega \rightarrow \infty$. Значит, в случае, когда $T \gg 1$, параметр $2 \gamma$ может быть определен как $1 / D$, где $D$ - размерность, вычисленная по формуле Ципфа, $T /(\gamma \alpha)$ - префактор [1].

\section{СПИСОК ЦИТИРОВАННОЙ ЛИТЕРАТУРЫ}

[1] Б. Мандельброт, Фрактальная геометрия природы, Ин-т компьютерных исследований, М., 2002. [2] V. P. Maslov, Russ. J. Math. Phys., 12:4 (2005), 483-488. [3] Л. Д. Ландау, Е. М. Лифшиц, Статистическая физика, Наука, М., 1964. [4] В. П. Маслов, Матем. заметки, 80:2 (2006), 220-230. [5] V.P. Maslov, Russ. J. Math. Phys., 13:3 (2006). [6] В.П. Маслов, Квантовая экономика, Наука, М., 2005. [7] В.П. Маслов, Матем. заметки, 78:3 (2005), 377-395. [8] С. М. Гусейн-Заде, Проблемы передачи информации, 24:4 (1988), 102-107.

\section{В. П. Маслов}

Поступило

Московский государственный университет

08.08.2006

им. М. В. Ломоносова

E-mail: v.p.maslov@mail.ru 